Noname manuscript No.

(will be inserted by the editor)

\title{
Selective Realism and the framework/interaction distinction: a Taxonomy of Fundamental Physical Theories
}

\section{Federico Benitez}

February 4, 2020

\begin{abstract}
Following the proposal of a new kind of selective structural realism that uses as a basis the distinction between framework and interaction theories, this work discusses relevant applications in fundamental physics. An ontology for the different entities and properties of well-known theories is thus consistently built. The case of classical field theories -including General Relativity as a classical theory of gravitation- is examined in detail, as well as the implications of the classification scheme for issues of realism in Quantum Mechanics. These applications also shed light on the different range of applicability of the ontic and epistemic versions of structural realism.
\end{abstract}

Keywords Scientific Realism • Philosophy of Modern Physics · Theory Classification

\section{Introduction: Framework and Interaction Theories}

It has recently been argued that a consistent version of selective realism should take into account the ontological level of the difference between what have been called interaction, as opposed to framework, theories. This classification is a refinement proposed by Flores (1999) to the classical distinction made by Einstein between principle and constructive theories (Einstein, 1919).

When I refer to theories I am mostly assuming a semantic view on theory structure (e.g. Suppes, 2002; Van Fraassen, 1989); thus, physical theories are identified as classes of mathematical models. More precisely, theories are identified with abstract theory-structures (families of mathematical models), standing in mapping relations to phenomena. For the scientific realist, the issue is then to give a consistent description of this mapping between models and phenomena that allows to consider the entities, properties, or laws in the

University of Lausanne, Department of Philosophy

E-mail: federico.benitez@unil.ch 
theories to be elements of objective reality, even on the face of the historical fact that theories have been superseded once and again. A strategy for this is to be selective at the time of assigning ontological commitments to different theories and pieces of theories (see Psillos, 2005). Such a take on scientific realism, and how it applies to known theories, is the leading thread of this work. This being said, the general notion of an interaction/framework division, which is the basis of this version of selective realism, is compatible with all the most known views on scientific theories, from the 'received view' of positivism (e.g. Hempel, 1970), to more modern pragmatist views (e.g. Cartwright, 1999).

The newer classification scheme for theories proposed by Flores (1999) uses a functional criterion: interaction theories are those that deal with the different way that entities are observed to interact (or somehow affect each other) in the world, whereas framework theories provide general constraints and common regularities for (generally more than one) interaction theories. A rich example of how this classification works can be studied at the level of Newtonian mechanics: Newton's three laws of motion give a regulative framework for the study of any force law, and should be seen as the overarching or background structure of classical mechanics. Conversely, specific force laws, such as the law of universal gravitation, deal with the details of one particular type of interaction, and work within this general framework. More modern examples of the usefulness of the classification are the building blocks of what follows.

The crux of the argument (starting from Romero-Maltrana et al. (2018)) is that the ontological commitment that should be attached to the different entities, laws, and properties within each of these two kinds of theories is in principle very different, and has not been properly discussed in the literature, which in my opinion sometimes leads to confusing metaphysical postures. This has implications for the ontology of a series of well-known theories from fundamental physics, an exploration of which is the aim of the present article.

Let me introduce a quick restating of the different ontological commitments that one can justify while taking as a basis the framework/interaction theory distinction. The distinction is especially useful when performing a case by case analysis of the ontological commitment due to entities, properties, and laws belonging to a given theory. In following the ontological facet of the framework/interaction classification, and always within the overarching theme of adopting a selective scientific realism to deal with the pessimistic metainduction, different kinds of structural realism are seen to be consistent with each type of theory.

The choice of structural realism (Worrall, 1989) as the preferred solution for the problem of the relation between scientific realism and theory change in science is spelled out in more detail elsewhere. The basic idea behind it is that even though scientific theories (and in particular fundamental physical theories) have been discarded and superseded throughout history, we can be confident given previous examples that there is something within the structure of present day theories that will be preserved in any future theory. In this way one can explain why science enjoys such an undeniable empirical success, while at the same time allowing for theory change. 
The version of structural realism first presented by Worrall (1989) came to be called epistemic structural realism (ESR). It states that our ontological commitment should be put not on the specific entities of scientific theories, but on their being part of an interaction structure, given that entities have shown to be less resilient to theory change. A more extreme version, known as ontic structural realism (OSR) (Ladyman and Ross, 2007; French, 2014), argues than in fact modern science proves that structure is effectively all there $i s$ to reality. The exact meaning of this provocative phrase changes a bit from author to author. Here I prefer the metaphysically consistent version exposed by (Esfeld and Lam, 2008; Esfeld, 2013), where structural relations join 'empty' relata: entities without any intrinsic property, that serve only as nodes to the web of relations.

A summary of my proposal would run like this: first, to follow the ideas of ESR when speaking about entities in interaction theories, which presuppose the existence of some real entity taking part in the relevant interaction. Conversely, the 'empty', only relational, entities of OSR provide a good match for framework theories, which are based on general principles and can be seen as purely structural in nature.

Besides this, general laws are more properly seen as the natural domain of framework theories; and framework laws should take some kind of ontological precedence over interaction laws. Looking for a more precise statement of this idea I introduce the notion of the relative modal strength of a realist position (details of which are presented elsewhere). The basic idea is that framework theories, serving as general constraints to any and all interaction theories embedded within, are seen as having a greater degree of necessity in a modal scale, as compared with the regularities or laws stemming from interaction theories. This is useful as well to understand the relative greater rigidity of framework laws on times of theory change.

Lastly, I propose a division of properties by means of the local/global axis, which I deem is more useful -within a structural approach- than the better known intrinsic/relational division. With this I propose that local properties are the natural domain of interaction theories, while global properties adjust themselves better to framework theories. Accordingly, I propose a greater ontological commitment to global properties in framework theories, and to local properties in interaction ones, by way of property translation if necessary (e.g. symmetry principles to local conservation laws in interaction theories).

This general scheme of selective structural realism is as good as its ability to deal with real-life scientific theories, as well as with the known history of theory change. I believe that the explanatory power of this approach is indeed greater than that of competing views, as can be seen in the examples from fundamental physics below. The examples also show how the specifics of each theory have to also be taken into account, on top of the previous considerations, when taking ontological commitments. 


\section{Classical Field Theory}

Beyond the relatively clear-cut case of Newtonian mechanics as explored elsewhere, it is interesting to study what these ideas imply in the case of field theories in the classical context. The most relevant example would be Maxwell's electrodynamics, clearly a theory of the interaction type, studying a specific type of interaction. It is interesting to note that the framework theory within which Maxwell's theory fits naturally is special relativity, even though the development of this interaction theory predates Einstein's work. In fact, were it not for the fact that electrodynamics is incompatible with the framework of classical mechanics, special relativity would not have had a reason to be developed at the turn of the twentieth century. As such, the framework/interaction divide is ahistorical, in the sense that, in principle, a given framework can be developed before, after, or at the same time as the interaction theories it naturally embeds.

Being an interaction theory, and following the previous discussion, one should choose a type of ESR with respect to the entities belonging to the theory. Therein lays a roadblock, as far at it is not completely clear which entities are to be taken as the fundamental entities for the theory. This problem repeats itself in the case of more modern theories, as will be shown below. In the particular case of Maxwell's theory, there are are two natural candidates for fundamental entities: (point) charges, and the electromagnetic field.

The standard textbook answer to this question would be that both charges and fields are needed in classical electromagnetism, and that its ontology should consider both. This received view notwithstanding, it is interesting to explore the issue with a little more depth, as has been done recently e.g. in Lazarovici (2016). Indeed, one could argue that the notion of a field is secondary, ontologically speaking, to the notion of a charged particle. In this view, fields would be useful mathematical concoctions, describing the way charges interact with each other, but having no intrinsic reality. This is in particular compatible with the Feynman-Wheeler absorber theory for the electromagnetic interaction (Wheeler and Feynman, 1949). Moreover, an ontology of both fields and particles also has to deal with the unsolved issue of the self-interaction of particles with the field they themselves generate, a problem that still lacks a fully satisfactory solution within the classical context.

The usual justification for the reality of the electromagnetic field is related to locality in special relativity. That is, as opposed to the case of the gravitational field in Newtonian gravitation, the electromagnetic field is to be reified because the interaction between charged particles is not instantaneous, but must obey the principles of special relativity (that is, the framework). This notion is contaminated by old ideas about the electromagnetic field as being the vibration of some kind of material substance. If, as is the case today, we think of fields either as substances in themselves or as properties of space-time points, this notion of locality preservation loses some of its weight, and is not so clear that it is to be preferred to the notion of unmediated action-at-a-distance with a retarded interaction (mathematically described by the electromagnetic 
field), were it not for physicist's historical averseness to this concept. As a famous example, Newton himself wrote that

It is inconceivable that inanimate Matter should, without the Mediation of something else, which is not material, operate upon, and affect other matter without mutual Contact... That Gravity should be innate, inherent and essential to Matter, so that one body may act upon another at a distance thro' a Vacuum, without the Mediation of any thing else, by and through which their Action and Force may be conveyed from one to another, is to me so great an Absurdity that I believe no Man who has in philosophical Matters a competent Faculty of thinking can ever fall into it. (Newton, 1756)

In terms of the distinction interaction/framework, I argue that it makes sense to reify (in the ESR sense) the entities that suffer from the corresponding interaction effect. As the electromagnetic field itself does not have electric charge, its electromagnetic interaction is justifiably seen as being of a secondary nature. This is easier to see in the relativistic notation of the theory, which treats the electric and magnetic fields as two aspects of the same entity, the field strength $F$ (using the classical Maxwell's notation electric and magnetic fields could be considered as independent substances which interact with one another). In this way, one could argue for a view where charges would be the fundamental entities of classical electromagnetism, with fields playing a secondary, derivative role. This view add further weight to the already well fundamented view of Lazarovici (2016).

There are, however, two separate senses in which one can consider Maxwell's theory. The first is as the theory of the classical electromagnetic interaction, such as in the discussion up to this point; the second is as the simplest example of a gauge theory, possessing an Abelian $U(1)$ internal symmetry. In this classical gauge theory, the symmetry only makes full sense in terms of fields, and, as per the discussion above for interaction theories, this symmetry property should be adjudicated to some localized real entity within the theory. It could be argued that gauge symmetry is in an ultimate analysis strongly associated with local charge conservation, but even this view can only be expressed in terms of fields or their potentials. In particular, the relation between charge conservation and $U(1)$ symmetry that can be observed using Noether's theorem presupposes the existence of dynamical fields (Schwarzbach, 2010).

In fact, Maxwell's theory is a special case of more general theories in two senses: first, electromagnetism is a special case of a Yang-Mills gauge theory. It is important to notice that in non-Abelian Yang-Mills theories the fields do carry their own (group) charge. As these are interaction theories, our criteria for these entails ESR with respect to the Yang-Mills fields, and, in the 'limit' of an abelian gauge group, this would entail the reality of Maxwell's fields. Moreover, all these generalized theories are non-linear, as opposed to Maxwell's, and it is very unclear (and indeed unlikely) that a construction similar to that of Wheeler-Feynman can be applied to these cases, in order to make the fields disappear from the ontology. 
Secondly, electromagnetism is the classical limit of quantum electrodynamics (QED). I will discuss the ontology quantum field theory in detail elsewhere, but suffice it to say that even if the correct ontology for QED is one of quantum particles instead of fields, the electromagnetic field has an associated particle, the photon, which should also reasonably be considered as real. Notice that QED, as opposed to classical electromagnetism, is a non-linear theory, as can be easily seen by the fact that photons, although being neutral entities, can interact with each other, and hence violate superposition, by means of virtual particles. This non-linearity can be seen as the root of the QED solution of the self-interaction issue, similar in spirit to the Born-Infeld solution for the classical case: QED solves the self-interaction problem of particles with their fields in terms of mass renormalization within the renormalization group (and in an interesting turn of events, self interaction effects are considered in order to reproduce the famous Lamb shift (Weinberg, 1995)).

There are some possible caveats to this reasoning. (i) QED and Yang-Mills theories are extensions or variations of electromagnetism, and it could be the case that the ontology to be taken in each case is completely independent. This is of course possible, but one would like some sort of parsimony in assigning ontological weight to theories, without such kind of abrupt changes of reification. This is analogous to the well-known Bohmian argument to preserve an ontology of particles in the classical-to-quantum transition. (ii) Within QED, one could propose to reify only the fermionic particles, associated to matter, as opposed to the bosonic particles such as the photon, commonly called 'force carriers'. Once more, this argument does not hold in the case of non-abelian gauge Yang-Mills theories, of which QED is only a subsector in the standard model of particle physics, in case one takes the (interaction theory) property of charge to be connected with the entity ontology, as per the general discussion above. On top of that, an exclusive fermion ontology is at odds with the standard model mechanism of fermion mass generation: by means of interaction with the (electroweak) charged bosonic Higgs field. (iii) The classical limit of Yang-Mills theories is not realized in the actual world, which may be interpreted as a point against working with an ontology of these as classical theories. However, the fact is that this absence from the classical world is relatively well understood. First, most non-abelian gauge theories are expected to show the phenomenon of quantum confinement, such as is the case for quantum chromodynamics. Confinement does not allow for the macroscopic detectability of properties associated with the fundamental charges, and these theories are thus not expected to show at the classical macroscopic level. Secondly, in cases where confinement does not play a role, such as in the electroweak sector of the standard model, spontaneous symmetry breaking of the gauge symmetry can make the associated interaction to be unaccessible at the macroscopic level, such as is the case for the weak interaction in our actual world.

To conclude this example, classical electromagnetism, as an interaction theory, can justifiably be considered both as a theory of point charges with derivative (non reified) fields, but without a clear metaphysical continuity to more modern theories; or like a theory of real point charges and fields 
(modulo the self-interaction issue, which should be seen as a quantum problem, somewhat alike to the singularity within a black hole in general relativity). In the latter case, arguably the most compatible with the structural realist answer to the issue of theory change, the relevant real properties are electrical charges and their fields as responsible of the gauge symmetry; while laws of e.g. charge and energy conservation, are expected to hold within the framework laws of special relativity, having to comply e.g. with the associated Lorentz invariance.

\section{Special and General Relativity}

Next, it is interesting to study the case of both special and general relativity, which I argue are to be considered of the framework kind. Of course, the philosophical interpretation of these theories is a research area in itself, and only a very succinct discussion of these topics is given here, with an emphasis on the ontological implications arising from the alleged framework character of these theories.

The matter seems quite clear for special relativity: indeed, this theory is one of the main examples in the original principle/constructive classification by Einstein. The dual principles of relativity of physics, and constancy of the speed of light, play the role of constraints on any possible physical process, with the electromagnetic interaction as described by Maxwell's equation being the historical focus. From these postulates many un-intuitive consequences follow, such as the relativity of simultaneity, and Lorentz contraction.

The constraints of special relativity can be expressed, as Minkowski found, as being equivalent to a particular structure of physical space and time (and therefore also of the causal relation). This space-time approach is also very clear in its framework character, serving as a literal background for any physical interaction.

Taking a closer look, the only entities one encounters in special relativity are the space-time points. As corresponds to a framework theory, I propose to give them at most only a completely relational notion of reality, as beckons to a moderate version of ontic structural realism, such as that proposed by Esfeld and Lam (2008). In this view, space-time substantialism cannot be justified from the principles of special relativity alone, although extra metaphysical information could in principle be used to take this view.

As an aside, there is an interesting connection with classical field theories. As described in the previous section, classical field theories are to be considered interaction theories within the framework of special relativity. Following the discussion above, it is a straightforward task to give an ontology for the fields of classical field theories: as opposed to space-time points, fields are entities of interacting theories, so a substantialism with respect to fields seems preferable over seeing fields as intrinsic properties of what i have argued should be considered as 'empty' space-time points. Again, space-time substantialism is still a valid metaphysical position, but cannot be justified by appeal to scientific realism alone in this selective realism framework. 
The natural properties and relations belonging to special relativity are the Lorentz invariants. In some sense all Lorentz invariant properties are global properties as I defined above: this invariance is something that is associated with the global transformations encoded in the Poincaré group (that is, translations, rotations, and boosts). It is interesting to note that already Lange (2001) argues that only Lorentz invariants should be seen as 'real' in a relativistic theory, being the only observer independent quantities.

Regarding laws or processes in special relativity, any interaction law within this framework has to follow the principles of Lorentz covariance. This strongly restricts the modal space for all such theories, as will be discussed further when considering quantum field theories in a forthcoming work. Of course, the rigidity at the time of theory change of this constraint is greater than that of any interaction theory. The theory change to general relativity (GR) is a case in point, in that the principle of equivalence ensures that the Minkowski structure of special relativity is preserved locally for every inertial observer, which is in fact the way by which interaction theories are introduced to GR (e.g. electromagnetism is translated from Minkowski to GR spacetime by the change from 'flat' space-time derivatives to covariant derivatives in Maxwell's field equations).

Turning to the case of GR, again one can define the theory by two postulates: the principle of equivalence and the principle of general covariance. Expressed in this way, it is clear that GR is a framework theory, constraining what physical processes are possible in our universe. As in the case of special relativity, the constraints of GR can be re-expressed in terms of a specific geometric structure for space-time. Following GR, the universe is a differentiable manifold, with the geometry of free-falling observers locally indistinguishable from Minkowski space-time. Within this framework structure, specific interaction theories, such as electromagnetism, introduce the mass-energy that dynamically modifies the space-time geometry following Einstein's field equations (Wald, 2010).

GR could naïvely be considered as a theory of the gravitational interaction, but the principle of equivalence does not allow for this. The insight by Einstein is that the equality between inertial and gravitational mass in Newton's theory implies that in GR there is no such thing as a gravitational interaction, only the effect of being within a non inertial frame of reference; that is, a geometric effect. GR stands thus as a particularly interesting theory form the point of view of the framework/interaction duality. In fact, GR can be seen as the first step within Einstein's effort to geometrize all of fundamental physics ${ }^{1}$. With this I mean the following: what would seem to be the natural playground of an interaction theory for the gravitational force was turned by Einstein, using the

1 Einstein himself might not have approved of this way of describing his program, see Lehmkuhl (2014). There, it is argued that Einstein himself considered GR to be a unification of gravity and inertia, and not a reduction of one into the other, and that his program was to find a further unity with electromagnetism. In the end, the result of the program would still be a framework theory (he would have called it a principle theory) where all of physics stands. 
equivalence principle, in a theory of non-inertial reference frames, and general covariance of physical laws: a framework theory. Later on, Einstein tried to further generalize this approach to electrodynamics, with the idea of arriving to a unified field theory which would have been a sort of framework 'theory of everything'.

This was also the ambition behind Wheeler's programme of geometrodynamics from the early sixties, which set out to geometrize away all interactions. Geometrodynamics (Wheeler, 1962) then was planned as a full framework theory of all of fundamental physics. Naturally, there are not any objects apart from space-time points in geometrodynamics, and it is unclear what interaction means when it is geometrized away, and what would be the ontological status of a theory without any interaction. As is well known, neither Einstein's nor Wheeler's efforts were successful. Much more can be said about the relationship between this 'geometrization' approach and the framework/interaction duality, but I leave further discussion of this for future work.

As before, the entities of a space-time theory such as GR are the space-time points. Suiting its framework character, these are naturally 'empty' of any intrinsic reality (Esfeld and Lam, 2008). This is in agreement with Einstein's solution to the hole argument, and once more against space-time substancialism. Again, this does not by itself disprove space-time substantialism, as it could possibly be justified using metaphysical arguments from outside the theory of GR.

As a side note, it is interesting to compare the framework character of space-time theories with the neo-Kantian point of view of e.g. Friedman (2014). In this vein, an argument can be built about all framework theories working as a kind of a priori setting for the physics of interactions. The analogies are strong, and to a certain point all structural views can be associated with some kind of neo-Kantian view, but this leads beyond the scope of this work. I leave open this line of inquiry for future investigations.

Finally, the discussion would be incomplete without mentioning that there is also an approach, first developed by Feynman, that recovers the equations of GR within a theory of a spin-2 field on a flat Minkowski space-time. This field gives the basis for the notion of the 'graviton' particle responsible for the gravitational interaction in some proposed theories of quantum gravity. In this case, one deals with a regular field theory for gravity, and in particular, with an interaction instead of a framework theory. Indeed, the natural framework for this spin-2 interaction theory of gravity is still special relativity. Even though both approaches recover the same field equations, the difference between the framework GR and the interaction spin-2 theory of gravity is of course fundamental, and each serves as a very different starting point in the search of a full theory of quantum gravity. 


\section{Shape Dynamics}

Another, perhaps less known example of a framework theory can be given in what Barbour (2012) calls Shape Dynamics (SD). Shape dynamics represents a modern, consistent take on Leibniz's notions of a purely relational space. Within SD, the relevant physical information about particle systems does not rest on their positions with respect to a background space, and how they change during an external independent time, but on their relative positions and their change thereof, i.e. ignoring any space and time scale external to those directly derived from the dynamics of matter itself.

It is worth noting that Barbour's shape dynamics has been successfully generalized as a theory of gravity that implements Mach's principle, developed with the goal to solve the problem of time in quantum gravity. Shape dynamics is dynamically equivalent to the canonical formulation of general relativity (in particular the ADM formalism, as shown by Gomes et al. (2011)). In more detail: it is equivalent to GR if this latter is limited to space -time geometries allowing for a $3+1$ foliation (which is the case for all physically relevant GR solutions). Given that this proposal is less well-known than GR, it is interesting to dedicate some paragraphs to describe its most salient features.

The main constrain of a relationalist geometry is the absence of a fixed unit of length. A moment of reflection allows one to visualize that, barring any external length scale or absolute reference system, two configurations of points showing the same overall shape would be indistinguishable from one another. That is to say, for example, that for a configuration of three particles, all similar triangles cannot be distinguished; therefore, these would count as identical configurations. Alternatively stated, the shape made by the point particles is what defines the state of the system. Notice though that SD includes as a primitive fact the known dimensionality of space.

What is known as shape space is the key concept of the proposal. Consider a configuration $q=\left(q_{1}, \ldots, q_{N}\right)$ of $N$ point particles and then translate all particles in the configuration in a given direction by the same amount: according to $\mathrm{SD}, q$ and its translated configuration $q^{\mathcal{T}}$ represent the very same physical situation, and, thus, they are identical. Suppose now to rotate $q$ : the new configuration $q^{\mathcal{R}}$ preserves the shape of the initial $q$, and consequently also $q$ and $q^{\mathcal{R}}$ are equivalent. Moreover, suppose we take into account a configuration $q^{\mathcal{S}}$ which differs from $q$ only by dilations (contractions or expansions, i.e. a scaling transformation) preserving its shape. Also $q^{\mathcal{S}}$, according to SD, will count as equivalent with respect to the initial configuration $q$.

Using a more technical jargon, in the case of a configuration of $N$ identical particles, shape space is constructed through a succession of identifications starting from the $3 N$-dimensional Cartesian configuration space $\mathcal{Q}^{N}$ for the configuration at hand. The first step to construct shape space consists in claiming that all the configurations that are carried into each other by translations $t \in \mathcal{T}$, the group of 3 dimensional Euclidean translations, represent the same shape and are not to be distinguished. Thus, $\mathcal{T}$ decomposes $\mathcal{Q}^{N}$ into its group orbits, which are defined to be the points of the $3 N-3$ dimensional quotient 
space $\mathcal{T}^{N}:=\mathcal{Q}^{N} / \mathcal{T}$. This first quotienting to $\mathcal{T}^{N}$ is straightforward. More significant is the second, which involves the 3 -dimensional rotation group $\mathcal{R}$ in order to construct the $3 N-6$ dimensional relative configuration space $\mathcal{Q}^{N}$ $:=\mathcal{Q}^{N} / \mathcal{T} \mathcal{R}$. Finally, one has to take into account the final quotienting by the dilatation (scaling) group $\mathcal{S}$, depending on one scale parameter, which leads to the shape space $\mathcal{Q}_{\text {Shape }}^{N}:=\mathcal{Q}^{N} / \mathcal{T} \mathcal{R S}$ of dimension $3 N-7$ (Barbour and Bertotti, 1982; Barbour, 2003, 2012). The groups $\mathcal{T}$ and $\mathcal{R}$ together form the Euclidean group, while the inclusion of $\mathcal{S}$ yields the similarity group.

The whole construction formalizes the fundamental geometrical notions of congruence and similarity. Two figures are congruent if they can be brought to exact overlap by a combination of translations and rotations and similar if dilatations are allowed as well. If we have a configuration $q$ of $N$ identical particles in Euclidean space, $q \in \mathcal{Q}^{N}$, we can 'move it around' with $\mathcal{T}$ or $\mathcal{R}$ or 'change its size' with $\mathcal{S}$, and the configuration would be always the same according to shape dynamics.

Once in possession of shape space, the history of a given configuration is then defined as a trajectory within it. Importantly, it is this evolution itself which yields a notion of 'passage of time', or better stated, a natural notion of duration. In the same way as there not being any external absolute ruler to measure positions, there is no external clock beyond the dynamics of the system. The easiest manner to visualize this notion in the classical case is by finding the geodesic flow of shapes in shape space, in terms of the metric inherited from the original Euclidean space. It is immediate to notice that these geodesics are parametrization invariant (exactly as in GR), with the natural definition of time corresponding to the choice of parameter that makes Newton's second law true (Barbour, 2012). As it has been shown in this reference, for a system of particles the time increment so defined takes the following form:

$$
\delta t=\sqrt{\frac{\sum_{i} m_{i} \delta x_{i} \cdot \delta x_{i}}{2(E-V)}},
$$

where $m_{i}, x_{i}$ represent the particles' masses and (Euclidean) positions respectively, whereas $E$ and $V$ are the classical total and potential energies. Notably, the time interval depends on the position change of every particle in the universe. As Barbour (2012) states

This is the first example of the holism of relational dynamics: the time that we take to flow locally everywhere is a distillation of all the changes everywhere in the universe. Since everything in the universe interacts with everything else, every difference must be taken into account to obtain the exact measure of time. The universe is its own clock.

There is certainly holism at work, as the full many-particle shape and its changes give the natural units of time and distance needed to interpret properties which are traditionally associated to each individual particle. This emergent notion of time is in line with Mach's ideas. Indeed, in his book The Science of Mechanics, Mach eloquently claimed: 
[...] we must not forget that all things in the world are connected with one another and depend on one another, and that we ourselves and all our thoughts are also a part of nature. It is utterly beyond our power to measure the changes of things by time. Quite the contrary, time is an abstraction, at which we arrive by means of the change of things; made because we are not restricted to any one definite measure, all being interconnected (Mach, 1960).

Shape Dynamics (as an ongoing program) is very tantalising in that it recovers the intuitions behind the Machian critique of Newtonian mechanics in a way that makes it compatible with the corroborated predictions of GR (Gomes et al., 2011) while at the same time allowing for some approximate notion of simultaneity or of space as decoupled form time to be brought back from the dustbin of theoretical physics. The theory would obviously serve as a framework for interaction theories, a framework which would dispense with non-relational surplus information. All interaction theories are by definition relational, and SD stands as a promising framework for further developments in physics, e.g. in the search for a quantum theory of gravity.

\section{Quantum Mechanics}

As a fourth application it is important to see what these ideas imply in the case of quantum mechanics (QM). First, it should be relatively clear from our characterization of theories that QM is to be considered a framework theory. Indeed, it postulates constraints and properties that are expected to be obeyed by any physical system. Examples of these postulates include the Born rule (and more generally the fact that the outcomes of measurements are in general probabilistic), and the Hilbert space structure valid for the description quantum states. Within this general framework which constrains the behaviour of any physical system one uses interaction theories to describe specific systems, such as e.g. electromagnetism to describe atoms. The topdown framework structure of QM can most easily be appreciated in the diverse approaches aiming for an axiomatic (Moretti et al., 2018) or informational theoretical (Clifton et al., 2003) expression of the theory.

Here it is important to emphasize what I understand is QM as a physical theory. One of the first motivations for the development of the semantic view on physical theories (Suppes, 2002) was ? demonstration that two radically different quantum-mechanic formalisms, the matrix and the wavefunction formalisms, were describing the same theory. This did not seem compatible with the at the moment dominating syntactic view on theory structure. Thus, QM is a class of mathematical models all of which which include the above mentioned characteristics (Hilbert space structure ${ }^{2}$, Born rule, etc). This is compounded by the existence of many so-called interpretations of QM, theories that add to the basic QM formalism in a variety of ways in order to solve (or dissolve)

${ }^{2}$ Or more precisely an underlying $C^{*}$-algebra structure. 
some of the issues that the bare formalism posses, such as the well-known measurement problem. What I contend here is that the framework character of QM is shared among all these models and interpretations. Importantly, this framework character should be independent of the specific interpretation given to the quantum wavefunction, or of which of the many possible solutions to the measurement problem is considered correct, if any.

However, the framework character view could be contested within some of the interpretations of QM. This dissonance may be mainly caused by previous attempts to use Einstein's distinction between constructive vs principle theories to the case of QM. Indeed, this is one of the cases where the framework/interaction divide is a clear improvement in clarity over the older view. For my purposes here, the distinction depends on whether QM works by providing constraints to the physics of any interaction, as opposed to modelling some particular interaction. For this it is useful to set apart for a moment the collapse postulate. Doing so, it is clear that standard textbook QM (and on reflection, any other interpretation before measurement happens) consists of a certain set of constraints over what is physically possible: probabilities instead of certainties about observables, Schrödinger evolution, etc. When interactions are treated in QM (for example using the quantum Hamiltonian operator) they are always interactions of some specific type, belonging to a specific interaction theory, e.g. electrodynamics.

Turning now to the collapse (or lack of it) of the wavefunction, certain interpretations could be seen to imply the existence of a new kind of purely 'quantum interaction', so that the framework character of QM could be challenged. Even within the standard von Neumann collapse postulate, the collapse is assumed to happen due to certain interactions with macroscopic measurement devices. However, in this (metaphysically unclear) standard case, such an interaction is always considered to be one of the already known interactions of the world: for example, the collapse of the electron wavefunction would be due to the electromagnetic interaction with a conducting measurement device. In the modern versions of Bohmian or Everettian interpretations there are no such extra interactions, as everything is governed by the Hamiltonian of the system and eventually that of the environment (these Hamiltonians usually include interactions terms belonging to different interaction theories). Conversely, in some other interpretations, such as Penrose's (Penrose, 2000), and arguably also in the Montevideo interpretation (Gambini et al., 2011), this extra interaction would be associated with quantum gravity effects.

The case of GRW collapse interpretations is different (e.g. Ghirardi et al., 1986, 1995). Within some versions of these, there can be an argument that there is a new, purely quantum interaction, responsible for the spontaneous collapse of the quantum wavefunction. If GRW interpretations turn out to be true, I would propose to divide the theory of QM into two different theories: one constraining the physics of any possible interaction, which I would call QM, and a second one, which might be called quantum self-dynamics, describing this new type of purely quantum interaction behind collapse. This solution, of separating the framework aspects from QM from the possible interacting 
aspects, would be also useful in the aforementioned cases where an interaction quantum theory of gravity should be used to understand collapse: gravity would be the necessary interaction theory.

To summarize, the top down character of QM, and the way it constraints the possible states of affairs in the world, imply its being a framework theory. Within the framework of QM one can study the quantum physics of specific (usually microscopic) systems, such as atoms, molecules, crystals, etc. This physics is described in terms of specific interaction theories, the most relevant of which is electromagnetism considered at the (semi-)classical level. Thus, the theory of the hydrogen atom sits within the framework of quantum mechanics, and involves the electric and magnetic classical interactions, together with classically known charges and masses for the proton and electron, as well as the purely quantum mechanical concept of spin (itself a postulated property).

This being the case, the approach I propose implies adopting (at best) an ontic form of structural realism for the entities in QM. The wavefunction in particular should be considered as real only inasmuch as its relational properties are involved, such as being compatible with the superposition principle. This does not necessarily mean that interpretations that are more strongly realist with respect to the wavefunction are to be discarded, only that this realism should be justified otherwise, not relying solely on the structure of QM. On top of that, as corresponds to a framework, the probabilistic nature of QM should be seen as very rigid with respect to theory change, and local quantum properties such as spin should better be seen as consequences of corresponding global properties, stemming from the postulates of the theory.

The case of spin is particularly interesting, because it is a purely relativistic effect appearing even at the level of non-relativistic QM due to its experimental implications. This fact can only be justified a posteriori, within a relativistic quantum theory, but the property of spin is a well-known consequence of Lorentz invariance and the Wigner representation theorem (Weinberg, 1995). Thus, one deals here with a deficient framework theory, that of Newtonian space-time, which forces on spin (and on related concepts, such as the spin-statistics relation) the need to be assumed as an extra postulate in non-relativistic QM in order to explain observations.

In two recent articles, Wallace $(2016,2018)$ also speaks of QM in terms of it being a 'framework' theory. The precise meaning of his nomenclature is not exactly the same as in this work, although there is a family resemblance of sorts. Wallace argues that QM should be seen not as a single theory, but as a class of theories, each related to a specific aspect of the quantum world: e.g. atomic physics as opposed to quantum computing. In my view, there is only one QM, but within this framework one can model the physics of many particular systems, using specific interaction laws. In this, the different approaches do not seem to differ in substance.

However, Wallace also uses this classification to declare that only the manyworld interpretation of QM is compatible with this framework character. First, he argues that non-representational interpretations, such as Copenhagen's, or QBism, fail to go beyond "explanations of features of quantum theory in the 
abstract", that is, to go beyond of the framework of QM, making "no use of details of any particular quantum system". In my alternative view, this is a necessary consequence of the fact that QM is a framework theory; and different arguments should be used instead to discard such interpretations. Second, Wallace makes a distinction between what he calls 'abstract' QM, and 'quantum particle mechanics', this last being QM expressed exclusively in position space instead of within an abstract Hilbert space. He then argues that Bohmian mechanics and GRW-type collapse interpretations cannot account for the applications of QM which are not given in terms of quantum particle mechanics, and therefore do not provide a good interpretation for the full QM framework. This line of reasoning does not work, because (i) both Bohmian mechanics and GRW theories have been shown to account for each known case of a specific quantum system which is not in principle represented by quantum particle mechanics (e.g. spin systems, or molecular systems (Norsen, 2014; Ghirardi et al., 1986)). (ii) Each interaction theory can and in fact generally does add some formalism to the framework theory (e.g. fields in classical electromagnetism), so the addition of non-spatial degrees of freedom to the framework of QM does not necessarily mean that QM itself must be non-spatial.

Indeed, as is argued by Esfeld (2013), assuming OSR for QM (as I propose for framework theories) does not suffice to justify preference of any interpretation of QM over the rest, as there are a number of extra 'for free' metaphysical assumptions that one can always make to accommodate any interpretation. Thus, taking an OSR stance does not help in solving the measurement problem of QM. Moreover, the fact that QM is a framework theory indicates that the solution to this indeterminacy with respect to interpretations should not be sought within the QM formalism, but instead in the study of particular interactions, with the hope of finding indications of incompatibilities with the standard framework, allowing for an exit to the measurement problem. Promising areas to look for incompatibilities are quantum computing, and perhaps relativistic QM and quantum gravity: the fact that the QM formalism is based on Newton's absolute time makes any time-related property of the theory (such as wavefunction collapse) ill-fated. Otherwise, it is always possible to use metaphysical arguments to choose the most promising interpretation of QM, but these arguments are usually not seen as decisive.

Nonetheless, we can imagine a first-order analysis of the different ontological status given to quantum entities by some of the most popular interpretations of QM. From the point of view of this work, it would seem ill-posed to try and give an ultimate ontological weight to the entities in QM. Thus, the many world interpretation, with its emphasis on the strict reality of the quantum wavefunction of a physical system (and beyond that, the alleged reality of an universal wavefunction describing the quantum state of all there is), seems to assign ontological commitments much farther that what would be warranted by a framework theory. Ontological commitments to entities should better come from interaction theories, such as electromagnetism. This can arguably be considered the case in Bohmian approaches, when the reality of point par- 
ticles is justified by by arguments outside of the characteristic of QM, and involving theories of e.g. electromagnetism. Conversely, approaches that put an emphasis on the instrumental role played by the wavefunction can and often do forget to take into account the ultimate reality of the physical system under study in each case: the existence of the electron as an entity participating in the electromagnetic interaction is more than reasonably well established, no matter what ontologic character is given to its quantum wavefunction.

Finally, as a very important aside, notice that some of the most relevant motivations for the radical take on structural realism of OSR are in fact quantum phenomena, such as entanglement and the quantum statistics of identical particles (French and Redhead, 1988; Ladyman and Ross, 2007; French, 2014). These phenomena follow from the postulates of the formalism, and the fact that e.g. all electrons are indistinguishable depends on our specific theory of electrons (QED), and not on QM as such. In any case, given that all the compelling physical reasoning behind OSR is done using examples from framework theories, it is easy to distrust the OSR position when dealing with interaction theories, a posteriori confirming the choices made in part I of this series. In fact, the other well-known justifications for OSR come from the argued lack of primitive thisness of space-time points, and from mathematical properties of fields within quantum field theory (QFT); and those entities also belong to framework theories (the case of QFT is discussed in the next part of this series, but suffice to say that its building blocks, namely SR and QM, are both framework theories). Thus the framework vs interaction distinction allows us to conceptually clarify the appeal and the limitations of OSR as justified from fundamental physics.

In conclusion, the fact that QM is a framework theory tells us that (i) its entities, such as the wavefunction, should be seen as having no intrinsic reality. (ii) The usual justifications for OSR only apply in cases like this, which is reasonable in retrospect. (iii) As such, the OSR position does not yield a 'natural' interpretation for QM. (iv) The (postulated) existence of local framework properties such as spin, together with the measurement problem, speak of the ultimate awkwardness of this framework theory, which should be superseded, at a minimum in accounting for a non-classical space-time structure.

\section{Conclusions}

In this work, I explore the implications of a recently proposed version selective scientific realism on a series of important fundamental physics theories. This realism stands on the notion that there are of two kinds of (at least fundamental) scientific theories. On the one hand there are theories that serve as frameworks, constraining what states of affairs or properties are possible in the world, and on the other hand there are interaction theories, dealing with the observed (and inferred) interactions between entities in the empirically ac- 
cessible world. This distinction, I contend, must have ontological implications, and provides a useful guide for the selective realist.

The examples of fundamental physics presented here show that it is always necessary to study the details of each framework or interaction theory when building this type of selective scientific ontology of the world. Thus, specific information coming from e.g. consistency conditions of the theory, or about the observability of its entities or properties, may have to be used to decide ontological commitment beyond only the theory type. An additional difficulty is that, as a scientific realist, one inevitably has to do with non-final, non well-defined, or otherwise incomplete theories. In these contexts, the selective realist should follow, I think, the most accepted consensus stemming from the scientific community.

The build up of this work leads to a notion of selective realism generically giving more weight to the ontological status of entities belonging to interaction theories, in particular in cases where the framework at hand can be considered incomplete or in some other way unsatisfactory.

Regarding the specific theories discussed above, some (possibly controversial) points deserve further emphasis. First, general relativity, although ostensibly a theory of the gravitational interaction, is in fact a framework theory. This is due to the fact that the whole construction of GR dismisses gravity as an interaction, turning it into a geometric effect instead. This is done by the principle of equivalence, one of the two general principles upon which the framework stands. Although highly non-trivial by itself, GR serves only as a framework for other kinds of interactions such as the electromagnetic or nuclear forces. This poses doubts on the ontological status of the relativistic space-time, and, consistently with my treatment of framework entities, I argue against a straightforward substantialist take on GR space-time.

Secondly, quantum mechanics is argued to also be a theory of the framework type, providing constraints on what can be called the space of possible physical processes. So, no matter what version or interpretation of QM one uses, measurement results must be probabilistic, with probabilities given by the Born rule, and physical states following a Hilbert space structure, etc, with all these constraints acting upon any physical system. This QM framework is occupied by diverse interaction theories, e.g. electromagnetism for the purposes of studying atomic or molecular physics.

Third, and more generally, the question of which version of structural realism, epistemic or ontic, is to be preferred, seems to be clearly connected to the character of the physical theory in question. Indeed, proponents of OSR justify their views using examples from modern physical theories which are, without exception, framework theories: quantum mechanics, general relativity, and quantum field theory (whose status will be discussed further in a forthcoming work). I argue that the entities in these theories naturally don't have much intrinsic character (e.g. space-time points, individual entangled identical particles, and so on) as a consequence of their being of the framework kind. Conversely, entities belonging to interaction theories, such as point particles or 
charges, seem more difficult to discard from an ontology, given that the whole point of the theory is to describe their behaviour.

These ideas themselves should serve as a framework of sorts for future discussions. In particular, I don't pretend to have exhausted the ontological implications of the framework/interaction theory dichotomy, nor all the ways this classification can contribute for a solution of the pessimistic meta-induction. Neither I think that the specific examples treated above are beyond revision; although, once again, further investigation on these would demand in my view a deeper comprehension of the characteristics of each case.

\section{References}

Barbour, J. (2003). Scale-invariant gravity: particle dynamics. Classical and quantum gravity, 20(8):1543.

Barbour, J. (2012). Shape dynamics. an introduction. In Quantum field theory and gravity, pages 257-297. Springer.

Barbour, J. B. and Bertotti, B. (1982). Mach's principle and the structure of dynamical theories. Proc. R. Soc. Lond. A, 382(1783):295-306.

Cartwright, N. (1999). The dappled world: A study of the boundaries of science. Cambridge University Press.

Clifton, R., Bub, J., and Halvorson, H. (2003). Characterizing quantum theory in terms of information-theoretic constraints. Foundations of Physics, 33(11):1561-1591.

Einstein, A. (1919). Time, space, and gravitation. Times (London), pages $13-14$.

Esfeld, M. (2013). Ontic structural realism and the interpretation of quantum mechanics. European Journal for Philosophy of Science, 3(1):19-32.

Esfeld, M. and Lam, V. (2008). Moderate structural realism about space-time. Synthese, 160(1):27-46.

Flores, F. (1999). Einstein's theory of theories and types of theoretical explanation. International Studies in the Philosophy of Science, 13(2):123-134.

French, S. (2014). The structure of the world: Metaphysics and representation. OUP Oxford.

French, S. and Redhead, M. (1988). Quantum physics and the identity of indiscernibles. The British Journal for the Philosophy of Science, 39(2):233246 .

Friedman, M. (2014). Foundations of space-time theories: Relativistic physics and philosophy of science. Princeton university press.

Gambini, R., García-Pintos, L. P., and Pullin, J. (2011). An axiomatic formulation of the montevideo interpretation of quantum mechanics. Studies in History and Philosophy of Science Part B: Studies in History and Philosophy of Modern Physics, 42(4):256-263.

Ghirardi, G. C., Grassi, R., and Benatti, F. (1995). Describing the macroscopic world: closing the circle within the dynamical reduction program. Foundations of Physics, 25(1):5-38. 
Ghirardi, G. C., Rimini, A., and Weber, T. (1986). Unified dynamics for microscopic and macroscopic systems. Physical Review D, 34(2):470.

Gomes, H., Gryb, S., and Koslowski, T. (2011). Einstein gravity as a 3d conformally invariant theory. Classical and Quantum Gravity, 28(4):045005.

Hempel, C. G. (1970). On the" standard conception" of scientific theories.

Ladyman, J. and Ross, D. (2007). Every thing must go: Metaphysics naturalized.

Lange, M. (2001). The most famous equation. The Journal of philosophy, $98(5): 219-238$.

Lazarovici, D. (2016). Against fields. European Journal for Philosophy of Science, pages 1-26.

Lehmkuhl, D. (2014). Why einstein did not believe that general relativity geometrizes gravity. Studies in History and Philosophy of Science Part B: Studies in History and Philosophy of Modern Physics, 46:316-326.

Mach, E. (1960). The science of mechanics: A critical and historical account of its development. Open court publishing Company.

Moretti, V. et al. (2018). Spectral Theory and Quantum Mechanics: Mathematical Foundations of Quantum Theories, Symmetries and Introduction to the Algebraic Formulation. Springer.

Newton, I. (1756). Four Letters from Sir Isaac Newton to Doctor Bentley: Containing Some Arguments in Proof of a Deity. R. and J. Dodsley.

Norsen, T. (2014). The pilot-wave perspective on spin. American Journal of Physics, 82:337-348.

Penrose, R. (2000). Wavefunction collapse as a real gravitational effect. In Mathematical physics 2000, pages 266-282. World Scientific.

Psillos, S. (2005). Scientific realism: How science tracks truth. Routledge.

Romero-Maltrana, D., Benitez, F., and Soto, C. (2018). A proposal for a coherent ontology of fundamental entities. Foundations of Science, pages $1-13$.

Schwarzbach, Y. (2010). The noether theorems.

Suppes, P. (2002). Representation and invariance of scientific structures. CSLI publications Stanford.

Van Fraassen, B. C. (1989). Laws and symmetry. Oxford University Press.

Wald, R. M. (2010). General relativity. University of Chicago press.

Wallace, D. (2016). Lessons from realistic physics for the metaphysics of quantum theory. Synthese, pages 1-16.

Wallace, D. (2018). Quantum theory as a framework, and its implications for the quantum measurement problem. Scientific realism and the quantum. Oxford: Oxford University Press.

Weinberg, S. (1995). The Quantum Theory of Fields, volume 1. Cambridge university press.

Wheeler, J. A. (1962). Geometrodynamics. Academic Press.

Wheeler, J. A. and Feynman, R. P. (1949). Classical electrodynamics in terms of direct interparticle action. Reviews of modern physics, 21(3):425.

Worrall, J. (1989). Structural realism: The best of both worlds?*. Dialectica, 43(1-2):99-124. 